\title{
MULTI-OBJECTIVE ENERGY-SAVING JOB-SHOP SCHEDULING BASED ON IMPROVED NSGA-II
}

\author{
Huo, D. X. ; Xiao, X. J.** \& Pan, Y. J.***\# \\ ${ }^{*}$ Department of Economics and Management, Xinzhou Teachers University, Xinzhou 034000, China \\ ** Jiangxi Police Institute, Nanchang 330103, China \\ *** Business College, Beijing Union University, Beijing 100025, China \\ E-Mail: yuejie.pan@buu.edu.cn $\left({ }^{\#}\right.$ Corresponding author $)$
}

\begin{abstract}
To pursue sustainable development, the manufacturing industry must meet strict requirements on energy-saving. However, the traditional manufacturing mode is not sufficiently green to satisfy such requirements. To solve the problem, this paper attempts to optimize the multi-objective energy-saving job-shop scheduling process. Firstly, a multi-objective optimization model was established to minimize the maximum makespan, total carbon emissions, and total tardiness. Then, the non-dominated sorting genetic algorithm II (NSGA-II) was improved to provide a solution to the multi-objective energy-saving job-shop scheduling problem (JSP). Finally, the effectiveness of the improved NSGA-II for solving the said problem was verified through simulation. The research provides a good reference for improving the greenness of manufacturing mode.

(Received in April 2020, accepted in July 2020. This paper was with the authors 2 months for 1 revision.)
\end{abstract}

Key Words: Job-Shop Scheduling Problem (JSP), Multi-Objective Energy-Saving Optimization, Non-Dominated Sorting Genetic Algorithm II (NSGA-II), Green Manufacturing

\section{INTRODUCTION}

Green manufacturing is an emerging production mode that meets the green demand of sustainable development. Under the mode of green manufacturing, job-shop scheduling needs to consider not only efficiency indices but also green indices, namely, energy consumption and carbon emissions $[1,2]$. The growing number of optimization indices inevitably complicates the solution to job-shop scheduling problem (JSP).

To save energy, the efficiency indices and green indices of the job-shop scheduling should be optimized in each production stage, creating a multi-objective energy-saving JSP. The objectives of the problem include improving production efficiency, shorten production cycle, eliminate the conflict between field resources, save energy, and reduce cost.

During job-shop production, scheduling determines the process route of each task, and defines the operation sequence and machines of every job. It is the most important link in the job production process, and the premise of job-shop scheduling. The selection of process route directly affects the quality, production time, and cost of each job [3].

In actual production, each job needs to be processed on multiple machines. There is a certain distance between the machines. The total makespan and distance vary with process routes. Besides, different process routes will lead to varied levels of carbon emissions. Therefore, the environmental impact of machines must be considered during the selection of the process route.

The distance between machines will also bring a certain amount of idle waiting time as a job is moved from one machine to another for subsequent processing. During the multiobjective energy-saving scheduling, it is possible for a machine to have no task for a long time. In this case, the machine needs to be shut down. The starting and shutdown periods of the machine also have an impact on the environment.

Therefore, this paper optimizes the multi-objective energy-saving job-shop scheduling process by improving the non-dominated sorting genetic algorithm II (NSGA-II), a novel 
swarm intelligence optimization algorithm, and verifies the effectiveness of the proposed method through simulation [4-6].

\section{LITERATURE REVIEW}

Considering various flexible factors in the manufacturing environment, many scholars have modelled the energy-saving JSP with multiple objectives (e.g. the makespan) and solved the model with new multi-objective energy-saving algorithms. For example, Yin et al. [7] established a multi-objective optimization model with high efficiency and low carbon, and proposed a compound solving method that transforms the problem into a single objective optimization problem through weight adjustment. To extend tool life and reduce energy cost, Luo et al. [8] modelled and minimized the total energy of job turning. Dai et al. [9] analysed the direct and indirect energy consumptions of operations and established the evaluation models for the two kinds of energy consumptions. Rose et al. [10] synthetized carbon emissions index, production efficiency index, and production benefit index into a carbon benefit index, and used it to compute the economic benefits per unit time and per unit carbon emissions. Jagadish, Ray [11] constructed a multi-objective green scheduling model, set up the sub-model for each objective, and put forward a green scheduling strategy based on the set of sub-models. Deng et al. [12] analysed the features of the production process, estimated the carbon emissions efficiency of process route, and maximized the efficiency with the non-dominated sorting genetic algorithm (NSGA).

Job-shop scheduling aims to achieve the optimization objectives by arranging the processing sequence of jobs on the machines according to the pre-set process route and determining the start and end times of each operation. To realize sustainable development, both green indices and efficiency indices should be considered in job-shop scheduling. As a result, the multi-objective energy-saving JSP has become a research hotspot. Vila et al. [13] explored the effects of energy-saving scheduling strategy on time and energy consumption, and developed a green genetic algorithm (GA) to solve the multi-objective energy-saving JSP, revealing that the use of low-energy machines can effectively reduce energy consumption. Yildirim and Mouzon [14] drew two important conclusions by analysing the relationship among energy consumption, processing time, and robustness: there are a clear correlation between robustness and energy consumption, and an obvious trade-off between robustness, energy consumption, and makespan. Lu et al. [15] designed a local search strategy to improve the local search performance of the GA. Moradi et al. [16] adopted to NSGA to solve the JSP with minimal energy consumption and total weighted tardiness. Zhang and Wu [17] created a jobshop scheduling model for machine energy consumption in different stages and solved the problem with simulated annealing (SA) algorithm. Lee et al. [18] constructed a job-shop scheduling model to optimize the energy consumption of machines in idle state and proved that minimizing that energy consumption helps to reduce the environmental impact of the production process. Gu et al. [19] designed an energy consumption model considering machine speed and presented a low-carbon scheduling algorithm considering makespan and energy consumption, aiming to solve flexible job-shop scheduling problem (FJSP). Akbari et al. [20] proposed a multi-objective bee colony algorithm (BCA) to improve the energy efficiency and production efficiency of the welding shop problem. Targeting the hybrid flow shop scheduling problem (HFSP), Khalili [21] put forward a multi-objective problem model with makespan, cost, and energy consumption as the optimization objectives, and improved the GA to solve the model. 


\section{MULTI-OBJECTIVE ENERGY-SAVING FJSP}

The scheduling process can be generally divided into three stages: feature sequencing, operation selection, and machine selection. The scheduling in the three stages could be highly flexible [22-25].

For convenience, the processing information of each job in the flexible job-shop scheduling is presented in Table I. The job has five processing features, each of which can be processed through one or several operations. Each operation needs to be executed on one of multiple available machines. The operations of a job can be expressed as a set $\left\{P_{1}, P_{2}, \ldots, P_{12}\right\}$, where $P_{i}$ is the $i^{\text {th }}$ operation.

Table I: The processing information of a job.

\begin{tabular}{|c|c|c|c|c|}
\hline Features & Operations & Optional machines & Processing time & Constraint \\
\hline$F 1$ & $P_{2}$ & $M_{1}, M_{3}$ & 3.4 & \\
\hline$F 2$ & $P_{3}-P_{5}$ & $M_{2}, M_{3} / M_{4}, M_{5}$ & $2,3 / 4,6$ & Before $F 5$ \\
\hline$F 3$ & $P_{6}-P_{8}$ & $M_{3}, M_{4}$ & 4,5 & Before $F 5$ \\
\hline$F 4$ & $P_{9}-P_{11}$ & $M_{1}, M_{2} / M_{3}, M_{5}$ & $2,4 / 3,6$ & \\
\hline$F 5$ & $P_{12}$ & $M_{5}, M_{6}$ & 4,5 & \\
\hline
\end{tabular}

\subsection{Problem description}

Scheduling aims to determine the sequence and machine for each operation. For the multiobjective energy-saving FJSP, two objectives need to be realized simultaneously: minimizing the total makespan and total carbon emissions. The following hypotheses were put forward for the problem:

(1) The machine for each operation is fixed.

(2) Each job is transmitted by an electric forklift between machines, and thus the energy consumption only depends on the transmission time.

(3) The carbon emissions are constant during the starting, preheating, and shutdown of each machine.

(4) If two adjacent operations are not executed on the same machine, and if the makespan exceeds the pre-set time, the current machine will be shut down.

(5) The operating power remains constant as an operation is being executed on a machine.

Based on the above hypotheses, the two objective functions can be established as:

- Minimizing the total makespan $f_{\min }(T)$

$$
f_{\min }(T)=P(T)+C(T)
$$

where, $P(T)$ is the processing time of each process; $C(T)$ is the transmission time of jobs on each machine.

$$
P(T)=\sum_{i=1}^{N} P\left(T_{i}\right)
$$

where, $N$ is the number of operations in the process route; $P\left(T_{i}\right)$ is the processing time of the $i^{\text {th }}$ operation in the process route.

If two adjacent operations in the process route are executed on different machines, the job must be transmitted from the machine of the current operation to that of the next operation. The transmission time can be calculated by:

$$
C(T)=\sum_{i=1}^{N-1} C\left(T_{M_{i}, M_{i+1}}\right) \times \delta\left(M_{i}, M_{i+1}\right)
$$

where, $M_{i}$ and $M_{i+1}$ are the machines for the $i^{\text {th }}$ and $(i+1)^{\text {th }}$ operations, respectively; $C\left(T_{M_{i}, M_{i+1}}\right)$ is the transmission time from $M_{i}$ and $M_{i+1}$.

If $M_{i} \neq M_{i+1}$, then $\delta\left(M_{i}, M_{i+1}\right)=1$; otherwise, $\delta\left(M_{i}, M_{i+1}\right)=0$. 
During job processing, the sources of carbon emissions include machines, cooler, and transport vehicle (i.e. the electric forklift). Hence, the objective function to minimize the total carbon emissions can be expressed as:

- Minimizing total carbon emissions $f_{\min }(C)$

$$
f_{\min }(C)=C(m)+C(\text { cold })+C(c v)
$$

where, $C(m), C(c o l d)$, and $C(c v)$ are the carbon emission of machines, cooler, and transport vehicle:

$$
C(m)=\alpha\left(E_{s}+E_{p}\right)
$$

where, $E_{s}$ and $E_{p}$ are the system energy consumption and load energy consumption of machines, respectively; $\alpha$ is the electric energy emissions factor.

$$
C(\text { cold })=\sum_{j=1}^{M} \frac{\sum_{i=1}^{N} P\left(T_{i j}\right) \times \delta_{i j}}{T_{j}} \times \alpha L_{i}
$$

where, $L_{i}$ is the fuel consumption of machine $i$ during job processing; $T_{j}$ is the fuel change cycle on machine $i$,

$$
C(c v)=P_{c v} \times \sum_{i=1}^{N-1} C\left(T_{M_{i}, M_{i+1}}\right) \times \delta\left(M_{i}, M_{i+1}\right) \alpha
$$

where, $P_{c v}$ is the transmission power of electric forklift.

\subsection{Problem solving by NSGA-II}

NSGA-II [26] is a fast non-dominated scheduling algorithm for multi-objective optimization problems. This subsection introduces how to solve multi-objective energy-saving FJSP with the NSGA-II as the optimizer. The solving process is illustrated in Fig. 1 below.

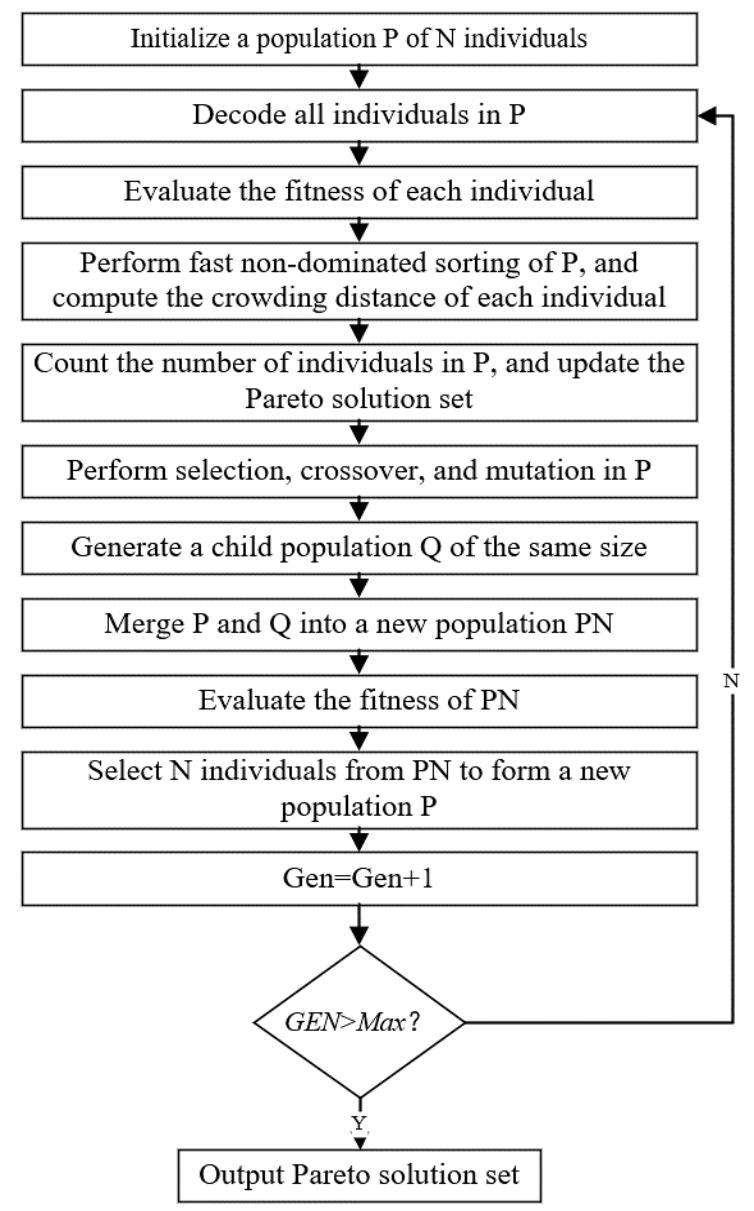

Figure 1: The workflow of solving multi-objective energy-saving FJSP with the NSGA-II. 
The solution space of the multi-objective energy-saving FJSP is treated as a population, in which each individual represents a scheduling plan. According to the multidimensional coding scheme, each individual contains a feature string, an operation string, and a machine string.

The feature string is initialized to adjust the sequence of features randomly. Of course, the feature sequence must satisfy the precedence constraints on different features. If the feature sequence is infeasible, it must be converted into a feasible sequence.

The operation string is initialized as follows: if feature $F_{n}$ is at the $k^{\text {th }}$ position of the job, there are $m$ optional operations. Then, random integers are generated between 1 and $m$, and inserted to the $k^{\text {th }}$ position of the operation sequence.

The machine string is initialized in a similar manner as the operation string.

The evaluation of individual fitness and selection are evaluated by two methods: fast nondominated sorting and crowding distance.

Each individual in the population is assigned a non-dominated rank through rapid nondominated sorting. For the population of $m$ individuals, it is assumed that $n_{i}$ individuals are dominated by the $i^{\text {th }}$ individual, and the individuals dominating others form a set $s i$. The process of rapid non-dominated sorting is as follows:

Step 1. Compare the fitness values of the individuals (fitness equals the sum of total makespan $T$ and total carbon emissions $C$ ), count the number of non-dominated individuals $q$ $\left(n_{i}=0, i \in\{1, \ldots, q\}\right)$, and set these individuals to level $1\left(i_{\text {rank }}=1\right)$.

Step 2. Determine the $s_{k}(k \in\{1, \ldots, q\})$ of each individual on level 1 , judge whether the $n_{t}-1$ of each individual $t$ in $s_{k}$ equals 0 , and set the individuals with $n_{k-1}=0$ to level 2 .

Step 3. Repeat Step 2 until all individuals are set to the corresponding rank.

Then, the individuals on the same level are subjected to fitness comparison based on crowding distance. The crowding distance of each individual is calculated as follows:

Step 1. Set the crowding distance $i_{d}$ of all individuals in the population to 0 .

Step 2. Rank the individuals in descending order of total makespan $T$, rank them again in descending order of total carbon emissions $C$, and set the crowding distance of boundary individuals as infinite. Then, the crowding distance of other individuals can be calculated by:

$$
i_{d}=\sum_{j=1}^{m}\left(\left|f_{j}^{i+1}-f_{j}^{i-1}\right|\right)
$$

where, $m$ is the total number of objective functions; $i_{d}$ is the crowding distance of the $i^{\text {th }}$ individual; $f_{j}^{i+1}$ and $f_{j}^{i-1}$ are the $j^{\text {th }}$ objective function values of the $(i+1)^{\text {th }}$ and $(i-1)^{\text {th }}$ individuals, respectively.

\section{OPTIMIZATION OF MULTI-OBJECTIVE ENERGY-SAVING JOB- SHOP SCHEDULING}

This section explores the multi-objective energy-saving FJSP and establishes a multi-objective energy-saving job-shop scheduling model, aiming to minimize the maximum makespan, total carbon emissions, and maximum total tardiness. In addition, the NSGA-II was improved to solve the established model.

\subsection{Multi-objective energy-saving job-shop scheduling model}

Multi-objective energy-saving job-shop scheduling can be described as follows: in a job-shop, $n$ jobs need to be processed on $m$ machines. Each machine has three working states: processing state, idle state, and adjustment state (during job change). The working state of each machine is greatly affected by the makespan of the jobs, the total carbon emissions of the process route, and other performance indices.

The following items are known in advance: the operations and operation sequence of each job; the machine and processing time of each operation; the energy consumption of each 
operation; the number of operations of all the jobs to be processed on each machine. However, the operation sequence on each machine is unknown.

Table II shows a multi-objective energy-saving FJSP with three jobs and three machines. The ultimate goal of the problem is to determine the operation sequence on each machine, and the starting time of each operation.

Table II: An example of multi-objective energy-saving FJSP.

\begin{tabular}{|c|c|c|c|}
\cline { 2 - 4 } \multicolumn{1}{c|}{} & \multicolumn{3}{c|}{ Machines (processing time, energy consumption) } \\
\hline Jobs & $O_{1}$ & $O_{2}$ & $O_{3}$ \\
\hline$J_{1}$ & $M_{2}(3,1.3)$ & $M_{1}(2,1.5)$ & $M_{3}(2,2.6)$ \\
\hline$J_{2}$ & $M_{3}(2,1.4)$ & $M_{2}(4,2.5)$ & $M_{1}(4,1.7)$ \\
\hline$J_{3}$ & $M_{1}(1,2.5)$ & $M_{2}(2,2.8)$ & $M_{3}(3,2.8)$ \\
\hline
\end{tabular}

In this paper, the multi-objective energy-saving job-shop scheduling needs to fulfil three objectives: minimizing maximum makespan $\left(T C_{\max }\right)$, minimizing total carbon emissions $\left(C E_{\max }\right)$, and minimize total tardiness $\left(T A_{\max }\right)$. The scheduling process must conform to the following hypotheses:

(1) The machine for each operation of a job is fixed.

(2) If adjacent operations on the same machine belong to the same job, there is no need for adjustment; if adjacent operations on the same machine belong to different jobs, it is necessary to make an adjustment.

(3) Once started, a machine cannot be shut down until completing all the operations assigned to it.

(4) The operating power remains constant as an operation is being executed on a machine.

(5) The same machine can only process one job at a time.

(6) Each job can only be processed on one machine at a time.

(7) The processing cannot be interrupted.

(8) There is no sequence constraint between operations.

Under the above hypotheses, the objective functions can be defined as:

(1) $f_{1}$ : Minimizing the maximum makespan

$$
\operatorname{Min} T C_{\max }=\left\{\max T C_{i}, i=1, \ldots, n\right\}
$$

where, $T C_{i}$ is the makespan of job $J_{i}$.

(2) $f_{2}$ : Minimizing total carbon emissions

During job processing, the carbon emissions can be divided into those generated from machines in processing state $C E_{p}$, those generated from machines in idle state $C E_{i}$, those generated from machines in adjustment state $C E_{f}$, and those generated from fuel consumption $C E_{c}$ :

$$
C E_{\text {max }}=C E_{p}+C E_{i}+C E_{f}+C E_{c}
$$

(3) $f_{3}$ : Minimizing total tardiness

To reduce the total tardiness of all jobs and improve consumer satisfaction, this objective function can be defined as:

$$
\operatorname{Min} T A_{\max }=\sum_{i=1}^{n} \max \left(0, T C_{i}-d_{i}\right)
$$

where, $d_{i}$ is the delivery date of job $J_{i}$.

The multi-objective energy-saving job-shop scheduling is constrained by the following factors:

(1) The operations of the same job must satisfy the following constraint:

$$
C T_{i, j}-P T_{i, j}+M\left(1-Y_{i}(h, j)\right) \geq C T_{i, h}, \quad i \in\{1,2, \ldots, n\}, j, h \in\{1,2, \ldots, m\}
$$

(2) The jobs on each machine must satisfy the following constraint: 


$$
C T_{i, j}-C T_{s, j}+M\left(1-X_{j}(i, s)\right) \geq P T_{i, j}, i, s \in\{1,2, \ldots, n\}, j \in\{1,2, \ldots, m\}
$$

(3) The job makespan must be greater than the sum of the starting time and processing time:

$$
C T_{i, j}>S T_{i, j}+P T_{i, j}, \quad i \in\{1,2, \ldots, n\}, j \in\{1,2, \ldots, m\}
$$

(4) The starting time of the current job on the machine must be greater than the sum of the makespan of the previous job and the adjustment time:

$$
S T_{k, j} \geq C T_{k-1, j}+t f_{k-1, k, j}, j \in\{1,2, \ldots, m\}
$$

where, $C T_{i, j}$ is the ending time of job $J_{i}$ on the $i^{\text {th }}$ machine; $S T_{i, j}$ is the starting time of job $J_{i}$ on the $j^{\text {th }}$ machine; $P T_{i, j}$ is the processing time of job $J_{i}$ on the $j^{\text {th }}$ machine; $M$ is a sufficiently large positive number; $Y_{i}(h, j)$ and $X_{j}(i, s)$ are decision variables.

\subsection{Improvement of the NSGA-II}

Considering the features of multi-objective energy-saving FJSP, this paper designs a local search strategy based on N5 neighbourhood structure and non-dominated sorting and relies on them to improve the NSGA-II to solve the problem.

Specifically, the process-based coding [27] was introduced to encode the individuals in the population. Let $n$ and $m$ be the number of jobs and machines in the multi-objective energysaving FJSP. Then, the chromosome is composed of $n \times m$ genes. The serial number of each job was defined based on the occurrence times of its gene in the chromosome.

For the problem in Table II, the gene sequence of one chromosome could be established as [ [ $\left.\begin{array}{llllllll}2 & 3 & 1 & 1 & 2 & 3 & 2 & 1\end{array}\right]$, where 1,2 , and 3 represent job $J_{1}$, job $J_{2}$, and job $J_{3}$, respectively. The values in the sequence indicate the processing sequence of the jobs.

In the NSGA-II, fast non-dominated sorting and crowding distance are adopted to evaluate the fitness of individuals in the population. The individual fitness is negatively correlated with the non-dominated level. For the individuals on the same level, their fitness values increase with the crowding distance.

The crossover of individuals was carried out by the POX method [28]. Let $P 1$ and $P 2$ be the parent chromosomes, and $C 1$ and $C 2$ be the child chromosomes after POX crossover. Then, the POX crossover can be realized through the following steps:

Step 1. Randomly divide all jobs into two non-empty subsets $S 1$ and $S 2$.

Step 2. Copy the jobs in $S 1$ of $P 1$ into $C 1$ and copy the jobs in $S 1$ of $P 2$ into $C 2$, without changing their gene positions.

Step 3. Copy the jobs in $S 2$ of $P 2$ into $C 1$ and copy the jobs in $S 2$ of $P 1$ into $C 2$, without changing their gene positions.

To enhance the local search ability of NSGA-II, this paper designs an individual local search strategy based on N5 neighbourhood structure and non-dominated sorting. The moving operation of $\mathrm{N} 5$ consists of two parts: the first part swaps the first operation and the subsequent operation of the machine; the second part swaps the last operation and the previous process of the machine. The first, last, previous, and subsequent operations of the machine are key blocks on the process route. Each part of the moving operation generates a new neighbourhood solution. The moving operation is explained in Fig. 2.

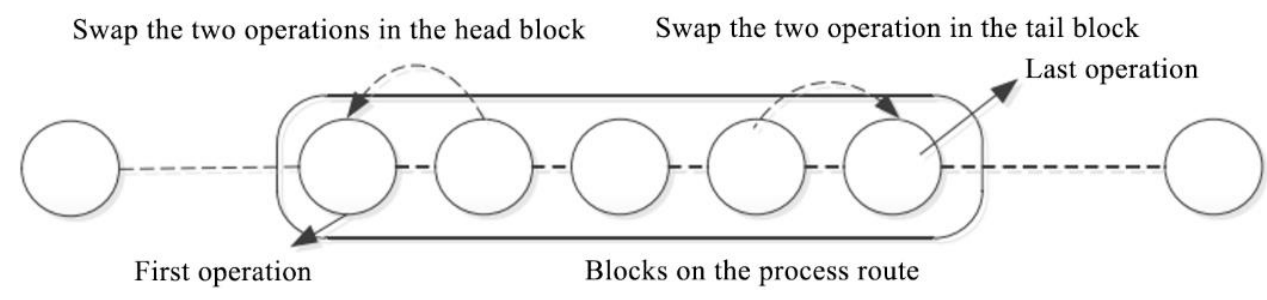

Figure 2: The moving operation of N5 neighbourhood structure. 
The proposed local search strategy combines non-dominated sorting method with N5 neighbourhood structure. By this strategy, the individuals meeting the multiple optimization objectives can be searched locally, and the non-dominated level of the current individual can be improved, making it possible to find more non-dominated solutions. The local search strategy can be implemented in the following steps:

Step 1. Count the total number of individuals in the current population $\left(p_{n}\right), i=1$, where $i$ is the serial number of the current individual.

Step 2. Generate the individuals $T_{i}$ in the neighbourhood of the current individual $i$ through the first moving operation of N5 neighbourhood structure.

Step 3. Decode each neighbourhood individual $T_{i}$ to obtain its objective function value. If the value is smaller than that of the current individual $i$, then neighbourhood individual $T_{i}$ dominates individual $i$. In this case, replace the neighbourhood individual $T_{i}$ with individual $i$. Otherwise, retain individual $i$.

Step 4. Generate the individuals $T_{i}$ in the neighbourhood of the current individual $i$ through the second moving operation of N5 neighbourhood structure.

Step 5. Decode each neighbourhood individual $T_{i}$ to obtain its objective function value. If the value is smaller than that of the current individual $i$, then neighbourhood individual $T_{i}$ dominates individual $i$. In this case, replace the neighbourhood individual $T_{i}$ with individual $i$. Otherwise, retain individual $i$.

Step 6. Perform $i=i+1$, and judge whether $i$ is greater than $p_{n}$. If yes, terminate the local search; otherwise, go back to Step 2.

\section{SIMULATION AND RESULTS ANALYSIS}

Due to the lack of benchmark test examples for multi-objective energy-saving FJSP, this paper designs ten groups of examples (Table III) for simulation and compares the effectiveness of the improved NSGA-II with the original NSGA-II. Table IV shows the adjustment times of each machine. Table V lists the simulation parameters of the improved NSGA-II.

Table III: The 10 groups of examples.

\begin{tabular}{|c|c|}
\hline Size & Jobs \\
\hline $4 \times 6$ & $J_{1}-J_{4}$ \\
\hline $6 \times 6$ & $J_{1}-J_{6}$ \\
\hline $10 \times 6$ & $J_{1}-J_{10}$ \\
\hline $20 \times 6$ & $J_{1}-J_{10}$ \\
\hline $6 \times 10$ & $J_{1}-J_{6}$ \\
\hline $10 \times 10$ & $J_{1}-J_{10}$ \\
\hline $15 \times 10$ & $J_{1}-J_{10}$ \\
\hline $20 \times 10$ & $J_{1}-J_{10}$ \\
\hline $25 \times 10$ & $J_{1}-J_{5}$ \\
\hline $25 \times 10$ & $J_{1}-J_{10}$ \\
\hline
\end{tabular}

Table IV: The adjustment times of each machine.

\begin{tabular}{|c|c|c|c|c|c|c|c|c|c|c|}
\hline$J_{0 b}$ & $\boldsymbol{J}_{\mathbf{1}}$ & $\boldsymbol{J}_{\mathbf{2}}$ & $\boldsymbol{J}_{\mathbf{3}}$ & $\boldsymbol{J}_{\mathbf{4}}$ & $\boldsymbol{J}_{\mathbf{5}}$ & $\boldsymbol{J}_{\mathbf{6}}$ & $\boldsymbol{J}_{\mathbf{7}}$ & $\boldsymbol{J}_{\mathbf{8}}$ & $\boldsymbol{J}_{\mathbf{9}}$ & $\boldsymbol{J}_{\mathbf{1 0}}$ \\
\hline $\boldsymbol{J}_{\mathbf{1}}$ & 0 & 3 & 4 & 3 & 2 & 2 & 4 & 3 & 2 & 3 \\
\hline $\boldsymbol{J}_{\mathbf{2}}$ & 2 & 0 & 4 & 3 & 2 & 2 & 4 & 3 & 2 & 3 \\
\hline $\boldsymbol{J}_{\mathbf{3}}$ & 2 & 4 & 0 & 3 & 2 & 2 & 4 & 3 & 2 & 3 \\
\hline $\boldsymbol{J}_{\mathbf{4}}$ & 2 & 4 & 6 & 0 & 2 & 2 & 4 & 3 & 2 & 3 \\
\hline $\boldsymbol{J}_{\mathbf{5}}$ & 2 & 4 & 6 & 3 & 0 & 2 & 4 & 3 & 2 & 3 \\
\hline $\boldsymbol{J}_{\mathbf{6}}$ & 2 & 4 & 6 & 3 & 2 & 0 & 4 & 3 & 2 & 3 \\
\hline $\boldsymbol{J}_{\mathbf{7}}$ & 2 & 4 & 6 & 3 & 2 & 1 & 0 & 3 & 2 & 3 \\
\hline $\boldsymbol{J}_{\mathbf{8}}$ & 2 & 4 & 6 & 3 & 2 & 1 & 3 & 0 & 2 & 3 \\
\hline $\boldsymbol{J}_{\mathbf{9}}$ & 2 & 4 & 6 & 3 & 2 & 1 & 3 & 5 & 0 & 3 \\
\hline $\boldsymbol{J}_{\mathbf{1 0}}$ & 2 & 4 & 6 & 3 & 2 & 1 & 3 & 5 & 2 & 0 \\
\hline
\end{tabular}


Table V: The simulation parameters of the improved NSGA-II.

\begin{tabular}{|l|c|}
\hline \multicolumn{1}{|c|}{ Parameters } & Value \\
\hline Initial population size $N$ & 200 \\
\hline Selection probability $p_{s}$ & 0.025 \\
\hline Crossover probability $p_{c}$ & 0.75 \\
\hline Mutation probability $p_{m}$ & 0.12 \\
\hline Pareto solution size & 30 \\
\hline Maximum number of iterations & 150 \\
\hline
\end{tabular}

The improved NSGA-II and NSGA-II were separately used to calculate the 10 groups of examples. For each group, the program was run independently for 50 times, and the Pareto solutions obtained by the two algorithms in the 50 independent simulations were counted, respectively. The number of Pareto solutions calculated by the two algorithms is shown in Table VI.

Table VI: The number of Pareto solutions calculated by the two algorithms.

\begin{tabular}{|c|c|c|}
\hline Size & Improved NSGA-II & NSGA-II \\
\hline $4 \times 6$ & 2 & 1 \\
\hline $6 \times 6$ & 17 & 8 \\
\hline $10 \times 6$ & 66 & 31 \\
\hline $20 \times 6$ & 58 & 46 \\
\hline $6 \times 10$ & 11 & 9 \\
\hline $10 \times 10$ & 33 & 27 \\
\hline $15 \times 10$ & 52 & 41 \\
\hline $20 \times 10$ & 57 & 52 \\
\hline $25 \times 10$ & 77 & 121 \\
\hline
\end{tabular}

Table VII: The sorted calculation results.

\begin{tabular}{|c|c|c|c|}
\hline Size & The number of Pareto solutions & Improved NSGA-II & NSGA-II \\
\hline $4 \times 6$ & 2 & 2 & 0 \\
\hline $6 \times 6$ & 8 & 6 & 2 \\
\hline $10 \times 6$ & 14 & 9 & 5 \\
\hline $20 \times 6$ & 56 & 55 & 1 \\
\hline $6 \times 10$ & 10 & 7 & 3 \\
\hline $10 \times 10$ & 31 & 29 & 2 \\
\hline $15 \times 10$ & 49 & 46 & 3 \\
\hline $20 \times 10$ & 52 & 50 & 22 \\
\hline $25 \times 10$ & 77 & 77 & 0 \\
\hline
\end{tabular}

Based on the data in Table VI, all Pareto solution sets obtained by the two algorithms for each group were combined, and the non-dominated solutions in the combined solution sets were counted (Table VII).

As shown in Table VII, the non-dominated solutions after sorting came from the solutions obtained by the improved NSGA-II, and most of the decomposition of the NSGA-II was dominated by the solutions of the improved NSGA-II. The Pareto solutions found by the improved NSGA-II had a higher non-dominated level than those found by the original NSGAII.

\section{CONCLUSIONS}

This paper mainly attempts to find a suitable solution to the multi-objective energy-saving FJSP. For this purpose, a multi-objective optimization model was established to minimize the maximum makespan, total carbon emissions, and total tardiness. Then, the NSGA-II was 
improved by designing an individual local search strategy based on N5 neighbourhood structure and non-dominated relationship. Ten groups of examples were created to test the improved NSGA-II and NSGA-II, respectively. The effectiveness of the improved NSGA-II in solving the multi-objective energy-saving FJSP is verified by comparing the results.

\section{ACKNOWLEDGEMENT}

This work is supported by Program for the Philosophy and Social Sciences Research of Higher Learning Institutions of Shanxi, China (No. 2020W156).

\section{REFERENCES}

[1] Seng, D. W.; Li, J. W.; Fang, X. J.; Zhang, X. F.; Chen, J. (2018). Low-carbon flexible job-shop scheduling based on improved nondominated sorting genetic algorithm-II, International Journal of Simulation Modelling, Vol. 17, No. 4, 712-723, doi:10.2507/IJSIMM17(4)CO18

[2] Li, D.; Liu, C. H.; Li, K. (2019). A remanufacturing logistics network model based on improved multi-objective ant colony optimization, Journal Européen des Systèmes Automatisés, Vol. 52, No. 4, 391-395, doi:10.18280/jesa.520409

[3] Gocken, T.; Dosdogru, A. T.; Boru, A.; Gocken, M. (2019). Integrating process plan and part routing using optimization via simulation approach, International Journal of Simulation Modelling, Vol. 18, No. 2, 254-266, doi:10.2507/IJSIMM18(2)470

[4] Jemilda, G.; Baulkani, S. (2018). Moving object detection and tracking using genetic algorithm enabled extreme learning machine, International Journal of Computers Communications \& Control, Vol. 13, No. 2, 162-174, doi:10.15837/ijccc.2018.2.3064

[5] Liu, Y. F.; Zhang, Q. S. (2018). Multi-objective production planning model for equipment manufacturing enterprises with multiple uncertainties in demand, Advances in Production Engineering \& Management, Vol. 13, No. 4, 429-441, doi:10.14743/apem2018.4.301

[6] Özdemir, H.; Sever, R.; Polat, Ö. (2019). GA-based optimization of SURF algorithm and realization based on Vivado-HLS, Traitement $d u$ Signal, Vol. 36, No. 5, 377-382, doi:10.18280/ts.360501

[7] Yin, L.; Li, X.; Gao, L.; Lu, C.; Zhang, Z. (2017). A novel mathematical model and multi-objective method for the low-carbon flexible job shop scheduling problem, Sustainable Computing: Informatics and Systems, Vol. 13, 15-30, doi:10.1016/j.suscom.2016.11.002

[8] Luo, X.; Goel, S.; Reuben, R. L. (2012). A quantitative assessment of nanometric machinability of major polytypes of single crystal silicon carbide, Journal of the European Ceramic Society, Vol. 32, No. 12, 3423-3434, doi:10.1016/j.jeurceramsoc.2012.04.016

[9] Dai, M.; Tang, D.; Giret, A.; Salido, M. A. (2019). Multi-objective optimization for energyefficient flexible job shop scheduling problem with transportation constraints, Robotics and Computer-Integrated Manufacturing, Vol. 59, 143-157, doi:10.1016/j.rcim.2019.04.006

[10] Rose, M. T.; Crossan, A. N.; Kennedy, I. R. (2008). Sustaining action and optimizing entropy: coupling efficiency for energy and the sustainability of global ecosystems, Bulletin of Science, Technology \& Society, Vol. 28, No. 3, 260-272, doi:10.1177/0270467608316097

[11] Jagadish, Ray, A. (2016). Optimization of process parameters of green electrical discharge machining using principal component analysis (PCA), The International Journal of Advanced Manufacturing Technology, Vol. 87, No. 5-8, 1299-1311, doi:10.1007/s00170-014-6372-8

[12] Deng, Z.; Lv, L.; Li, S.; Wan, L.; Liu, W.; Yan, C.; Zhang, H. (2016). Study on the model of high efficiency and low carbon for grinding parameters optimization and its application, Journal of Cleaner Production, Vol. 137, 1672-1681, doi:10.1016/j.jclepro.2016.03.022

[13] Vila, S.; Guirado, F.; Lerida, J. L.; Cores, F. (2019). Energy-saving scheduling on IaaS HPC cloud environments based on a multi-objective genetic algorithm, The Journal of Supercomputing, Vol. 75, No. 3, 1483-1495, doi:10.1007/s11227-018-2668-z

[14] Yildirim, M. B.; Mouzon, G. (2012). Single-machine sustainable production planning to minimize total energy consumption and total completion time using a multiple objective genetic algorithm, IEEE Transactions on Engineering Management, Vol. 59, No. 4, 585-597, doi:10.1109/ TEM.2011.2171055 
[15] Lu, P.-H.; Wu, M.-C.; Tan, H.; Peng, Y.-H.; Chen, C.-F. (2018). A genetic algorithm embedded with a concise chromosome representation for distributed and flexible job-shop scheduling problems, Journal of Intelligent Manufacturing, Vol. 29, No. 1, 19-34, doi:10.1007/s10845-015$1083-\mathrm{Z}$

[16] Moradi, H.; Zandieh, M.; Mahdavi, I. (2011). Non-dominated ranked genetic algorithm for a multiobjective mixed-model assembly line sequencing problem, International Journal of Production Research, Vol. 49, No. 12, 3479-3499, doi:10.1080/00207540903433882

[17] Zhang, R.; Wu, C. (2011). A simulated annealing algorithm based on block properties for the job shop scheduling problem with total weighted tardiness objective, Computers \& Operations Research, Vol. 38, No. 5, 854-867, doi:10.1016/j.cor.2010.09.014

[18] Lee, H.; Park, S.; Jeong, H. (2013). Evaluation of environmental impacts during chemical mechanical polishing (CMP) for sustainable manufacturing, Journal of Mechanical Science and Technology, Vol. 27, No. 2, 511-518, doi:10.1007/s12206-012-1241-6

[19] Gu, J.; Jiang, T.; Zhu, H.; Zhang, C. (2020). Low-carbon job shop scheduling problem with discrete genetic-grey wolf optimization algorithm, Journal of Advanced Manufacturing Systems, Vol. 19, No. 1, 1-14, doi:10.1142/S0219686720500018

[20] Akbari, R.; Hedayatzadeh, R.; Ziarati, K.; Hassanizadeh, B. (2012). A multi-objective artificial bee colony algorithm, Swarm and Evolutionary Computation, Vol. 2, 39-52, doi:10.1016/ j.swevo.2011.08.001

[21] Khalili, M. (2014). A multi-objective electromagnetism algorithm for a bi-objective hybrid no-wait flowshop scheduling problem, The International Journal of Advanced Manufacturing Technology, Vol. 70, No. 9-12, 1591-1601, doi:10.1007/s00170-013-5376-0

[22] Ikechukwu, A. J. (2019). Assessment of organizational performance of private manufacturing companies: the impact of supply chain management responsiveness, Journal of System and Management Sciences, Vol. 9, No. 3, 26-44, doi:10.33168/JSMS.2019.0302

[23] Simo, A.; Barbulescu, C.; Kilyeni, S.; Dragos, C. (2019). Manufacturing process monitoring in terms of energy management improving, International Journal of Computers Communications \& Control, Vol. 14, No. 3, 388-400, doi:10.15837/ijccc.2019.3.3560

[24] Mu, H. P. (2019). Disruption management of flexible job shop scheduling considering behavior perception and machine fault based on improved NSGA-II algorithm, Journal Européen des Systèmes Automatisés, Vol. 52, No. 2, 149-156, doi:10.18280/jesa.520206

[25] Ojstersek, R.; Lalic, D.; Buchmeister, B. (2019). A new method for mathematical and simulation modelling interactivity: a case study in flexible job shop scheduling, Advances in Production Engineering \& Management, Vol. 14, No. 4, 435-448, doi:10.14743/apem2019.4.339

[26] Sun, G.; Bin, S. (2018). A new opinion leaders detecting algorithm in multi-relationship online social networks, Multimedia Tools and Applications, Vol. 77, No. 4, 4295-4307, doi:10.1007/s11042-017-4766-y

[27] Li, W. D.; Ong, S. K.; Nee, A. Y. C. (2004). Optimization of process plans using a constraint-based tabu search approach, International Journal of Production Research, Vol. 42, No. 10, 1955-1985, doi:10.1080/00207540310001652897

[28] Zhao, S. (2015). Bilevel neighborhood search hybrid algorithm for the flexible job shop scheduling problem, Journal of Mechanical Engineering, Vol. 51, No. 14, 175-184, doi:10.3901/ $\underline{\text { JME.2015.14.175 }}$ 